\title{
President Thanks 1988 MRS Volunteers
}

The Materials Research Society has had a year of exceptional growth and solid achievement. In meeting today's needs and in creating the foundations for future progress, MRS has set dynamic records. Vital to these accomplishments is the energetic and inspired leadership provided by those members who have volunteered both time and expertise to serve the various MRS activities. MRS is fortunate in the dedication of all of its volunteers, and I want to thank all those who have served as committee members or chairs, or elsewhere in the whole spectrum of MRS activities. I would like especially to congratulate and thank those involved as leaders, whose names appear below. Their invaluable service has been a key to this most successful year for the Materials Research Society.

John E.E. Baglin, President

\section{Leadership Honors 1988 Officers}

R.P.H. Chang (1st Vice President) J.M. Phillips (Secretary)

\author{
K.C. Taylor (Immediate Past President) \\ P.S. Peercy (2nd Vice President) \\ S.M. Kelso (Treasurer)
}

\section{Councillors}

B.R. Appleton

R.W. Balluffi

David K. Biegelsen

Walter L. Brown

G. Slade Cargill, III (Fall)

David E. Clark (Spring)

W. Wade Adams

B.R. Appleton

Andrzej R. Badzian

Aaron Barkatt

David M. Barnett

C. Batich

Bertram Batlogg

S. Bernasek

E.E. Berry

D.K. Biegelsen

John C. Bravman

Clyde L. Briant

C. Jeffrey Brinker

Merton H. Brooks

W.L. Brown

Jean Bussiere

W.H. Butler

Greg Campbell

Don Capone

Irving J. Chabinsky

Hong K. Choi

Paul C.W. Chu

William A.T. Clark

John Clarke

Roy Clarke

George Van B. Cochran

Roy H. Cooper, Jr.

G. Dresselhaus

M.S. Dresselhaus

James Druzik

Russell Dupuis
D.E. Clark

\author{
R.P.H. Chang \\ Charles B. Duke \\ Rodney C. Ewing \\ Robert A. Huggins
}

Herbert H. Johnson

Noble M. Johnson

S. Thomas Picraux

John M. Poate
Della M. Roy

Rustum Roy

Frans Spaepen

C.W. White

\section{Meeting Chairs}

Clifton W. Draper (Spring)

D. Wayne Goodman (Fall)

C.T. Liu (Spring)

J. Francis Young (Fall)

\section{Symposium Chairs}

Lester Eastman
Ronald K. Eby
Alan Eckbreth
M. Endo
A.G. Evans
Larry Fehrenbacher
S.G. Fishman
Simon Foner
Michael W. Geis
Ronald Gibala
John Gland
R.J. Gottschall
Joe E. Greene
Mihal E. Gross
Y. Hamakawa
Kay Hays
R.I. Hemmings
John Holbrook
Robert A. Huggins
Robert Hull
Hiroshi Ishiwara
Joseph Jasinski
A. Wayne Johnson
George H. Johnson
B.H. Kear
J.A. Knapp
Kristine Knutson
C.C. Koch
Roger Koch
William Krakow
Robert Langer
Bennett C. Larson

P.G. LeComber

F.D. Lemkey

Robert W. Lenz

C.T. Liu

G.L. Loper

A. Madan

A. Madhukar

Benoit B. Mandelbrot

D.M. Mattox

G.J. McCarthy

Donald E. McLemore

Gholamabbas Nazri

Robert J. Nemanich

William D. Nix

Claudine Noel

R. Nuzzo

Charles M. Packer

D.E. Polk

Fernando A. Ponce

Larry E. Pope

Lynn E. Rehn

Lawrence R. Roberts

Rustum Roy

Manfred Ruhle

Devendra K. Sadana

Leonard M. Sander

Edward V. Sayre

David N. Seidman

Duward F. Shriver

Ronald A. Siegel

R.W. Siegel

T.W. Sigmon 


\section{Journal of Materials Research}

W.L. Brown (Editor in Chief)

\section{Principal Editors:}

$\begin{array}{ll}\text { R.W. Cahn } & \text { P.S. Peercy } \\ \text { M.S. Dresselhaus } & \text { R. Petkovic-Luton } \\ \text { W.L. Johnson } & \text { Rustum Roy } \\ \text { F.F. Lange } & \text { Shigeyuki Somiya } \\ \text { Werner Lutze } & \text { R.A. Street }\end{array}$

\section{Advisory Review Board:}

P. Chaudhari

M.E. Fine

H.C. Gatos

\author{
P.S. Peercy \\ Rustum Roy \\ Shigeyuki Somiya
}

P.B. Hirsch

R.A. Huggins
Clifford H. Griffiths (Indexer)

Robb Thomson

Takashi Tokuyama

David Turnbull

C.W. White

J.I. Nishizawa

W.P. Slichter

\section{MRS Bulletin}

E.N. Kaufmann (Editorial Chairman)

I.W. Boyd (Associate Editor - Europe)

\section{International Advisory Board:}

M. Balkanski

S. Hsu

\begin{abstract}
R. Krishnan
\end{abstract}
H.D. $\mathrm{Li}$

R. Roy

G.D.W. Smith

Technical Editorial Board:

J.C.C. Fan

F.Y. Fradin

G.L. Liedl

S. Namba

R.W. Schwoebel

R.C. Sundahl

\section{Committee/Subcommittee Chairs}

R.P.H. Chang

C.W. Draper

Charles B. Duke

J.P. Gambino

J.M. Gibson

Aubrey L. Helms, Jr.

\section{Harold Anderson}

Orlando Auciello

Sergio Ajuria Jessica M. Bailey Joseph A. Breindel Mildred S. Dresselhaus

John E. Fischer

G. Fischman

Jonathan A. Foreman

$\begin{array}{ll}\text { Carol M. Jantzen } & \text { J.M. Phillips } \\ \text { E.N. Kaufmann } & \text { Gordon E. Pike } \\ \text { Jeffrey A. Kelber } & \text { S. Thomas Picraux } \\ \text { W. Krakow } & \text { Peter P. Pronko } \\ \text { Vivienne Harwood Mattox } & \text { L. Michael Quick }\end{array}$

\section{Sections}

Michael Current Karel K. Czanderna

Michael I. Kelley

Anupam Madhukar

\section{Student Chapters}

Warren M. Garrison, Jr.

Theresa A. Guiton

Jeff Luster

R.K. MacCrone

John Hawley

Linn W. Hobbs

James D. Jarratt

Kevin S. Jones

Brenda Kamps
Martha L. Mecartney

Gary Messing

Carey A. Pico

Edward J.A. Pope

Steve Richards
J.M. White C.G. Willson J.M. Woodall Alex Zunger

J.B. Wachtman, Jr. F.W. Young, Jr.
T. Sugano

J.S. Williams

K.C. Taylor
J.B. Roberto Alton D. Romig Barry E. Scheetz Kathleen C. Taylor C.W. White

James L. Marshall

Rustum Roy James W. Taylor Michael O. Thompson David C. Van Aken Wendell Williams A.S. Yue

We warmly congratulate the victors in the 1988 MRS elections and wish them well as they take up their new, or renewed, responsibilities: Russell R. Chianelli, First Vice President and President-Elect; James B. Roberto, Second Vice President; Julia M. Phillips, Secretary; Susan M. Kelso, Treasurer; and Slade Cargill, Elton N. Kaufmann, Gordon E. Pike, and Kathleen C. Taylor, Councillors. 